\title{
The research of digital watermarking technology based on Neural Network
}

\author{
Liling Zhang \\ Dept of Computer Science and Engineering, Hubei University of Education, Wuhan, China,430205 \\ hu_tu@qq.com
}

\begin{abstract}
In the paper a kind of hyperchaos neural network is creatively used to realize the encryption of digital images. By selecting a reasonable initial value for the hyperchaos neural network, an encrypted matrix can be attained to encrypt the digital watermark; meanwhile, the chosen 3-layer wave division technique is adopted to realize image's encryption. The proposed method makes well use of the non-predictability of chaos system and the close dependence on initial value, as well as that the encrypted matrix cannot be got by illegitimate users. Thereto, the chosen 3-layer wave algorithm is also unknown to unauthorized users, which can powerfully protect the copyrights of digital images. The experiment results show that the method is sound and reasonable with great invisibility and robustness.
\end{abstract}

Key Words: hyperchaos neural network system, initial value, encrypted matrix; Watermark

\section{基于神经网络的数字水印技术研究}

\author{
章丽玲 \\ 湖北第二师范学院计算机科学与工程系, 武汉, 中国, 430205 \\ hu_tu@qq.com
}

摘要:神经网络系统是从人脑动力系统 中抽象出来的一类重要的人工神经计算模 型。将超混沌神经网络系统的特殊性引入到 数字水印领域来, 可有效实现数字图像的加 密。本文通过选择超混沌神经网络的合理初 始值, 得到一个加密矩阵进行水印的加密, 并结合三层选择性小波分解技术实现图像的 加密。该方法通过混沌系统的不可预测性以 及对初值的敏感依赖性来确保加密矩阵对非 法用户未知, 通过选择性三层小波解密算法 也是非法用户所无法获知的来有力保障了数 字图像的版权。实验研究表明, 该水印算法 具有很好的不可见性和鲁棒性。

关键词:超混沌神经网络系统; 初始值; 加密矩阵; 水印

\section{1 引言}

随着现代社会多媒体技术的飞速发展, 图片因为其包含的信息丰富, 而成为各行各 业不可缺少的工作对象。为了防止在图像传 输和共享过程中的侵权行为, 迫切需要行之 有效的加密技术来确保图像的所有权。
目前混沌的研究被广泛地应用于通信系 统的同步、解密技术中, 同时在信息的保密 通信方面也发挥着越来越重要的地位。将混 沌系统应用到图像加密与信息隐藏方面, 可 在知识产权保护等方面获得很广泛的应用。 因此, 本文创造性地运用了超混沌神经网络, 实现了数字图像的加密。其中 $\mathrm{s}$ 加密矩阵通 过选择超混沌神经网络的初始值得到, 初始 值的取值是无限的, 所以可以产生无限的 $\mathrm{s}$ 加密矩阵。正是因为 $\mathrm{s}$ 加密矩阵的无限性, 在提取水印的时候, 没有正确的 $\mathrm{s}$ 加密矩阵, 就无法正确提取水印, 从而有力保障了数字 图像的版权。在检测水印时, 只要有超混沌 神经网络初始值, 和图像频域十六分之一的 信息, 即可进行水印的提取。攻击实验研究 表明, 本文提出的算法, 具有很好的抗攻击 的能力以及具有很好的鲁棒性。

\section{2 超混沌神经网络系统的建立}

神经网络系统是从人脑动力系统中抽象 出来的一类重要的人工神经计算模型, 神经 网络可以用类似人脑的方式存储记忆和模 
式, 而且该模式可以在提供部分信息的情况 下重现。本文选择以下神经网络系统来进行 数字水印的加密:

$$
\begin{aligned}
& \dot{x}_{i}=-c_{i} x_{i}+\sum_{j=1}^{4} w_{i j} v_{j} \quad(i=1,2,3,4) \ldots \ldots \\
& v_{i}=f_{i}\left(x_{i}\right)
\end{aligned}
$$

其 中 , $f_{i}\left(x_{i}\right)=\left(\left|x_{i}+1\right|-\left|x_{i}-1\right|\right) 2 /$, $W=\left(w_{i j}\right)$ 是 $4 \times 4$ 的矩阵, 当 $\mid i-j p 1$ 时 $w_{i j}=0$, 选择参数 $c$ 与 $W$ 分别为:

$$
\begin{aligned}
c & =[1.1,1.5,0.4,125]^{T} \\
W & =\left(\begin{array}{cccc}
2.1 & 2.18 & 0 & 0 \\
-3.88 & 1 & 3.1109 & 0 \\
0 & -2.9 & 0.5 & -1.2 \\
0 & 0 & 100 & 160
\end{array}\right) \ldots \ldots
\end{aligned}
$$

为该神经网络系统选择一个初始点 $\mathrm{X}_{0}=[0.1601,0.1372,-0.1941,1.1342]^{\mathrm{T}}$ 时, 则可得到一个超混沌吸引子, 如图 1 所示。

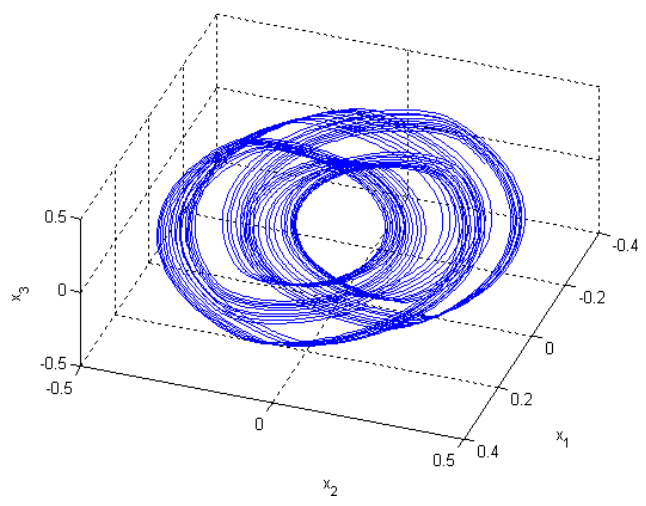

(a) 超混沌吸引子三维图 (x1:x2:x3)

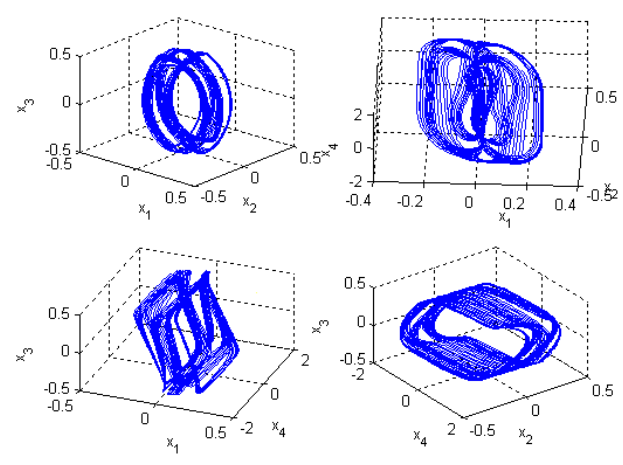

(b) 超混沌吸引子三维图

图 1 : 基于 $X 0=[-0.0705,-0.1685,0.0051,-1.2836] \mathrm{T}$ 的超 混沌吸引子

\section{3 基于超混沌神经网络的数字水印}

\section{1 嵌入水印算法描述}

在确定了混沌神经网络系统后, 可通过 选定初始值 $\mathrm{X}_{0}$, 利用已生成的混沌神经网络 系统, 产生序列 $X$, 进而获得 $32 \times 32$ 的矩阵 $\mathrm{s}$ 来对原始水印进行加密处理。其算法为:

$\mathrm{a}_{0}=\bmod (\mathrm{X}(1:$ end $-1,1) ， 2 ） ; / /$ 从序列 $\mathrm{X}$ 的第 1 列中获取数据

$\mathrm{a}_{1}=\bmod (\mathrm{X}(2$ : end 4$) ， 2) ; / /$ 从 序列 $\mathrm{X}$ 的第 4 列中获取数据

$\mathrm{a}=\mathrm{a}_{1}>\mathrm{a}_{0} ; / / /$ 比较 $\mathrm{a}_{0}$ 与 $\mathrm{a}_{1}$ 的大小, 若 $\mathrm{a}_{1}>\mathrm{a}_{0}$, 则 $\mathrm{a}=1$; 反之 $\mathrm{a}=0$

$\mathrm{W}_{1}=\mathrm{a}(1: 1024) ; / / /$ 取 $\mathrm{a}$ 的前 1024 个 数据构成一个 $1 \times 1024$ 矩阵

$\mathrm{s}=$ reshape $\left(\mathrm{W}_{1}, 32,32\right)$; //将 $\mathrm{W}_{1}$ 改造 成一个 $32 \times 32$ 矩阵。

该算法的描述为: 错位比较序列 $\mathrm{X}$ 第 2 维数据和第 3 维数据的大小, 得到逻辑数列 $\mathrm{a}$, 取 $\mathrm{a}$ 的 1024 个记录, 并将 $\mathrm{a}$ 变换成 $32 \times$ 32 的矩阵。

(1) 原始水印如图 2(a)所示; 利用 arnold 变化, 进行 4 次 arnold 操作, 对原始水印 $\mathrm{w}_{0}$ (32×32) 进行置乱, 得到 $w_{1}$, 如图 2(b) 所 示。

（2）利用超混沌神经网络, 产生得到 $32 \times 32$ 的矩阵 $\mathrm{s}$ 来对 $\mathrm{w}_{1}$ 进行加密处理, 产生 嵌入水印 $\mathrm{w}_{2}$, 即: $\mathrm{w}_{3}=\mathrm{w}_{1}+\mathrm{s}$, 如图 2(c)。

\section{乘就}

(a) 原始水印 $\mathrm{w} 0$

(b)置乱水印W1

(c) 嵌入水印 $\mathrm{w} 2$

图2: 利用超混沌神经网络系统对水印进行加密

(3) 对原始图像 I（256×256, 如图 3 所示) 进行三层小波分解（如图 4 所示）。 其中, 三层选择性小波分解步骤如下:

a) 第一层小波分解：分解原始图像 I, 得到系数块 LL1、LH1、HL1、HH1； 其中 LL1 为低频系数, HH1 为高频系 数, LH1 为右上角系数块, HL1 为左 下角系数块;

b) 第二层小波分解: 对 LH1 进行小波分 解, 得到系数块 LH11、LH12、LH13、 LH14; 对 HL1 进行小波分解, 得到 
系数块 HL11、HL12、HL13、HL14;

c) 第三层小波分解: 对 LH11 进行小波 分解, 得到系数块 H111、LH112、 LH113、LH114; 对 HL11 进行小波分 解, 得 到 系 数 HL111,HL112,HL113,HL114。同时, 记录系数块 LH112、LH113、HL112 以及 HL113。

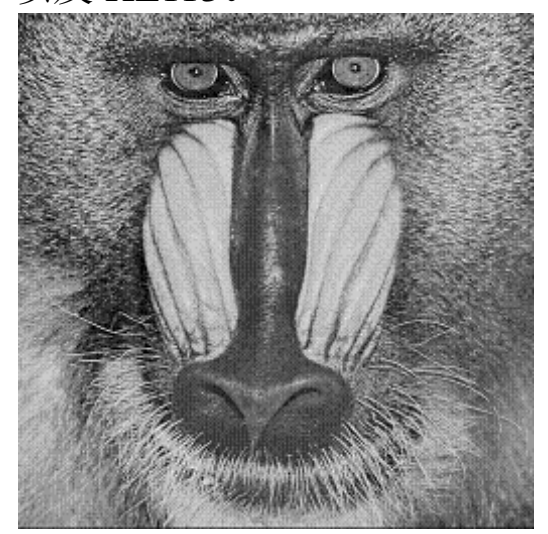

图 3: 原始图像

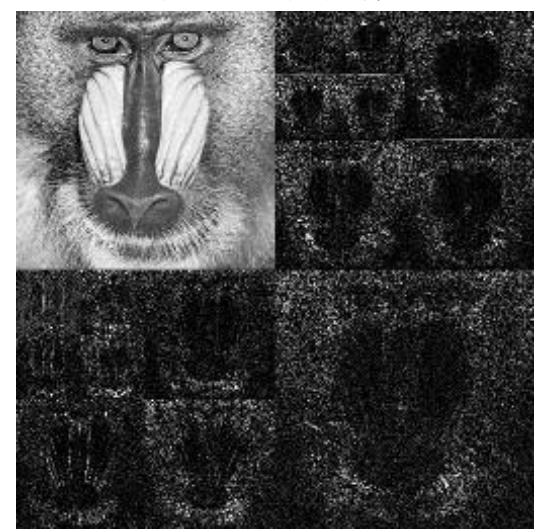

图 4: 基于三层小波分解的图像

（4）水印嵌入: 对 LH112、LH113、

HL112、HL113（32×32）分别进行水印嵌入, 步骤为:

a) 设定幅值 $b$;

b) 因 LH112、LH113、HL112、HL113 与 $w_{3}$ 均是 $32 \times 32$ 的, 它们的元素可以 一一对应起来。若水印对应位置上的 值为 0 ，则 LH112、LH113、HL112、 HL113 相应位置上的系数的幅值增加 $b$; 否则, 减少 $b$ 。

(5) 重建图像: 进行反小波变换, 重建 图像。如果选择 $b=10$, 则可得到重建图像如 图 5 所示。

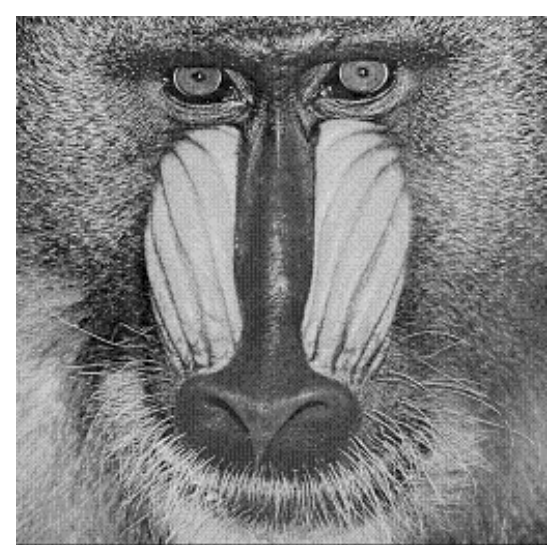

图 5: 含水印的图像

\section{2 水印的提取}

在水印嵌入算法的基础上, 水印提取算 法可描述为:

(1) 对待检测图像 $X_{1}$ 进行三层小波分 解, 提取相应位置的系数; 分别与保存的系 数块 LH112、LH113、HL112、HL113 进行比 较。比较方法如下:

$$
\begin{aligned}
& \operatorname{IF~K}(\mathrm{i}, \mathrm{j})>\mathrm{K}_{0}(\mathrm{I}, \mathrm{j}) \\
& \mathrm{W}(\mathrm{i}, \mathrm{j})=0 ; \\
& \text { ELSE } \\
& \quad \mathrm{W}(\mathrm{i}, \mathrm{j})=1 ; \\
& \text { END IF }
\end{aligned}
$$

其中 $\mathrm{K}$ 代表 $X_{1}$ 进行三层小波分解, 提取 相应位置的系数块; $K_{0}$ 可代表 LH112、 LH113、HL112、HL113（i，j=1，2，…, $32)$ 。

（2）综合 4 个位置提取的水印，设置阈 值, 判断得出水印 $w_{4}$ 。

（3）利用 $s$, 解密 $w_{4}$, 得到 $w_{5}$ 。

(4) 利用 arnold 变换的周期性, 得出最 终提取的水印 $w_{6}$ 。

\section{4 实验与结果分析}

为了检验实验效果，本章设计了相关的 攻击实验来对本文所提出的方法进行检测, 并通过通用的检出率来衡量检出效果, 其中 检出率为: $c=1-\frac{\sum_{i=1}^{32} \sum_{j=1}^{32}\left|w_{0}(i, j)-w_{6}(i, j)\right|}{32 \times 32}$ 。

含水印图像（图 5) 未受攻击时, 提取 的水印如图 6 所示, 此时提取的水印与原始 水印完全相同, 检出率为 1 。 


\section{冀豪}

图 6: 未受攻击时提取的水印

对含水印图像（图 5) 进行如图 7（a） 所示的规则剪切, 图 7 (b) 为提取的水印。 该规则剪切攻击的检出率为 0.9531 。

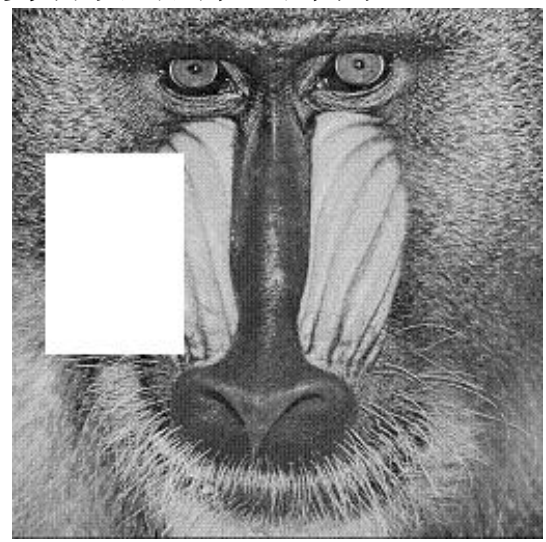

（a）经过规则剪切的水印图像

\section{恝意}

(b) 提取的水印

图 7: 基于规则剪切的水印图像实验

对含水印图像进行如图 8（a）所示的不 规则剪切, 图 8（b) 为相应提取的水印。该 不规则剪切攻击的检出率为 0.9463 。

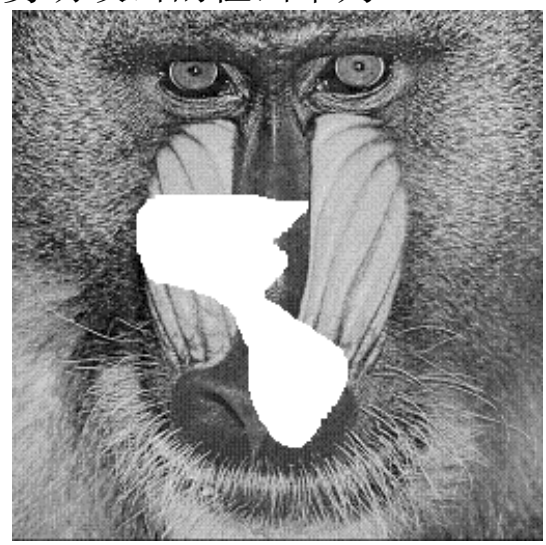

（a）经过不规则剪切的水印图像

\section{掏变}

(b) 提取的水印

图 8: 基于不规则剪切的水印图像实验

对含水印图像加入椒盐噪声(如图 9(a)) 的提取, 图 9（b) 为相应提取出的水印。基 于椒盐噪声攻击的检出率为 0.9170 。

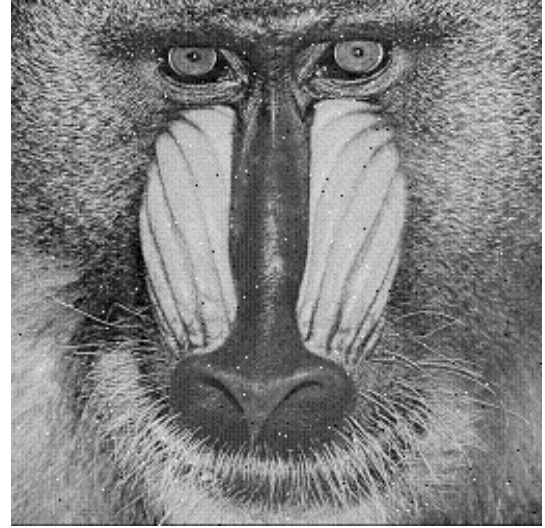

(a) 加入椒盐噪声的水印图像

\section{鼠变}

（b）提取的水印

图 9: 基于椒盐噪声的水印图像实验

对含水印图像进行 JPEG 压缩, 图像数 据量压缩为原来的 $33.93 \%$ ，如图 10（a）所 示, 提取的水印如图 10 (b) 所示。基于 JPEG 压缩的检出率为 0.9551 。

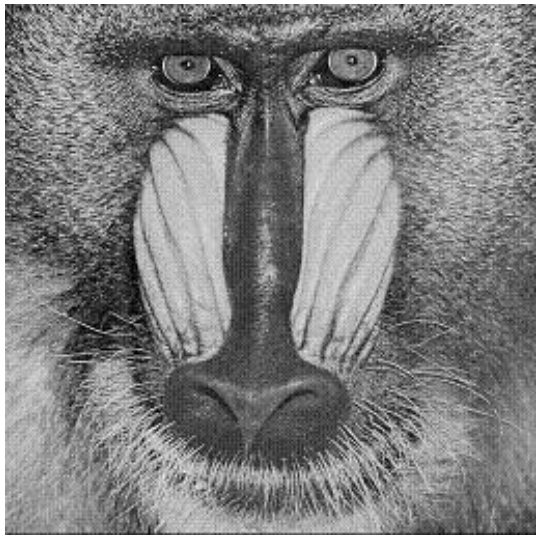

(a) 经过 JPEG 压缩的水印图像

\section{耄孪}

(b) 提取的水印

图 10: 基于 JPEG 压缩的水印图像实验

实验表明，本文所提出的超混沌神经网 络水印算法, 有效地利用了混沌系统的不可 预测性以及对初值的敏感依赖性，加强了水 印信息的保密性; 在水印嵌入图像以后，图 像的品质未受影响, 这表明本文的水印嵌入 算法具有很好的不可见性; 在含水印图像遭 受不同程度的压缩、规则剪切、不规则剪切、 模糊加噪等攻击后仍能有效检测到水印的存 在，且提取的水印具有很高的相似性，这表 明该超混沌水印算法具有很好的鲁棒性。 


\section{5 结论}

本文提出一种基于超混沌神经网络的数 字水印技术。在该方法中, 本文主要利用混 沌系统的不可预测性, 结合三层选择性的小 波分解技术进行图像的加密。因为混沌系统 的不可预测性和对初值的敏感依赖型, 加密 数据是非法用户无法得到的; 而选择性三层 小波解密算法也是非法用户所无法获知的, 从而有力保障了数字图像的版权。水印的检 测只需要图像频域十六分之一的信息, 在含 水印图像遭受不同程度的压缩、剪切、模糊 加噪等攻击后仍能有效地检测到水印的存 在。

\section{References}

[1] Huang Yan, Yang Xiao-Song. Horseshoes in a Class of Simple Circuits, Chaos, Solitons and Fractals. 2006, 29: 131-140.

[2] Huang Yan, Yang Xiao-Song. Horseshoes in Modified Chen's Attractors. Chaos, Solitons and Fractals, 2005, 26: 79-85.

[3] Huang Yan, Yang Xiao-Song. Horseshoe in a Class of Planar Mappings. Discrete Dynamics in Nature and Society. 2006, 10(1155): 1-10.

[4] Huang Yan, Yang Xiao-Song. Hyperchaos in a New Family of Simple CNN. International Journal of Bifurcation and Chaos. 2006, 11(16): 3341-3348. 